\title{
Understanding social decision-making from another species' perspective
}

\author{
Sarah F. Brosnan ${ }^{1}$
}

Published online: 3 November 2017

(C) Psychonomic Society, Inc. 2017

Summary One challenge of studying cognition and behavior in other species is designing studies that are intuitive and motivating to the subjects; studies that lack these features may result in false negatives and other outcomes that bias our understanding of animals' abilities and choices. Here, Schmelz, Grueneisen, Kabalak, Jost, and Tomasello (PNAS, 114(28), $7462-7467,2017)$ investigated prosocial behavior, in which animals may make decisions that benefit a conspecific, and found that, contrary to much earlier work, when chimpanzees are given a reason to do so, they do make prosocial choices.

Keywords Prosocial behavior - Chimpanzee $\cdot$ Experimental methodology $\cdot$ Reciprocity

Humans are not the only highly cooperative primate, but to what degree is human cooperation motivated by the same underlying psychological mechanisms that underpin cooperation in other primates? There are many proposed mechanisms, one of which is our propensity for prosocial behavior. Humans are strongly prosocial, making choices that benefit others, particularly in contexts in which the choice reciprocates an earlier benefit that one's partner provided. Evidence for such behavior in other primates remains mixed, including in chimpanzees, one of our closest phylogenetic relatives. Although the results from controlled lab studies have often failed to provide evidence of prosocial behavior in chimpanzees, scientists who have observed these animals for many years argue that they do witness prosocial behavior, and long-term datasets indicate

Sarah F. Brosnan

sarah.brosnan@gmail.com

1 Departments of Psychology, Philosophy \& Neuroscience, Center for Behavioral Neuroscience, and Language Research Center, Georgia State University, Atlanta, GA, USA reciprocation between social partners in well-established social groups. This lack of consistency between the experimental evidence and observations suggests that at least part of the discrepancy is due to experimental design: The tasks are intuitive to us, but may not be to them. It is also challenging to ascertain whether subjects understand the task and the accompanying payoff contingencies in the ways that we intend and are motivated to make choices in the ways we expect them to be.

In the typical prosocial experiment, a subject chooses between two distributions of food, one of which rewards only them, and the other of which rewards both them and their partner (typically equally, since it is presumed that prosocial behavior is more common in less costly situations). Scientists consider a result evidence of prosocial behavior if the subject chooses the option that rewards both individuals more often in a social condition in which the partner is present than in a solo condition in which there is no partner to receive the food (Jensen, Hare, Call, \& Tomasello, 2006; Silk et al., 2005). Although a few studies have shown evidence indicating prosocial choices, in many cases the subjects are indifferent, choosing both options equally often regardless of whether a partner is present. This remains the case even when the task is altered such that partners take turns being the subject that makes the decision, which was hypothesized to increase the potential benefits of choosing the prosocial option, via reciprocity.

This task is widely used, but it is not clear that subjects understand its contingencies and purpose. The former understanding has been easier to demonstrate; experiments typically include attention controls, such as trials in which only one of the two options rewards the subject, to ensure that the subjects have not simply learned that it doesn't matter what they choose, because they get rewarded either way. Most studies also run comprehension checks in which subjects have access to the outcomes of both options, to ensure that they prefer the "prosocial" option when it directly benefits them.

What has been harder to determine is whether subjects understand the purpose of the task, and/or are motivated to use it 
to provide a benefit to a member of their social group, as is the experimenter's intention. This is the critical question that all of us who do experimental work on animal behavior and cognition struggle with: Do the subjects understand our tasks in the way that we intend them to, or are our tasks so far removed from subjects' typical interactions as to be irrelevant? Indeed, one of the few studies to find evidence of prosocial behavior in chimpanzees seems to indicate that the subjects failed to comprehend the purpose of the original task. The subjects in this study were tested on the standard procedure and, as is typical, were indifferent between the options. Following this, they received experience in the partner role, with a stooge subject who had been trained to choose the prosocial option. When then repaired with the original partner, subjects were more likely to choose the prosocial option (Claidière et al., 2015). One potential explanation for these results is that the experience as a benefited partner helped the chimpanzees see the task in the way that the experimenters intended them to.

In this task, Schmelz, Grueneisen, Kabalak, Jost, and Tomasello (2017) also used the standard procedure, but with a tweak; the subject could only choose between the prosocial and selfish options if the partner first released a latch that allowed the subject access to the decision apparatus. In the first study, the partner had to give up immediate access to two pieces of food to give the subject access to the apparatus. The subject would then choose between the prosocial option, in which both subjects would get two pieces of food, and a selfish option, in which the subject would get two pieces but the partner would get nothing (unbeknownst to the subjects, the partner was a stooge trained to make the prosocial choice). Subjects' choices were compared to a no-assistance condition, in which the partner was present but the experimenter released the latch. Subjects were indifferent in the no-assistance condition $(50.7 \%$ prosocial choices), but chose the prosocial option almost $75 \%$ of the time when the partner gave up food to make the choice available. An additional study added a condition in which the partner did not need to give up food to release the latch. In this case, subjects chose the prosocial option $67.5 \%$ of the time, less often than when the partner had given up guaranteed food, but more often than in the no-assistance condition (53.5\% in this study). The experimenters ruled out order effects, indicating that this was not a result of increased experience with the apparatus.

Most impressive, the subjects also chose the prosocial option even when it was costly. In another study, the subjects faced the same apparatus, except that the selfish option included an additional piece of food, so the subject had to give up a piece of food to make the prosocial choice (in this study, there were always three pieces of food available, including in the alternate option for the partner, except for the selfish option, which had four). Nonetheless, although prosocial choices dropped to $17.4 \%$ in the no-assistance condition, subjects continued to choose the prosocial option $43.8 \%$ of the time when their partner gave up a guaranteed three pieces of food to release the latch.
Why was this this study successful, whereas so many others had failed to find evidence of prosocial behavior? For one thing, the experimenters gave the subjects extensive experience with the apparatus prior to the study and utilized strict criteria to determine which subjects understood the contingencies of the task. Subjects who did not meet the criteria were dropped prior to the experiment. Although this halved the sample size, such pretesting eliminates concerns about post-hoc determinations of "reliable" subjects while also avoiding false negatives due to subjects who did not understand the task. Moreover, Schmelz et al. (2017) gave the subjects a reason to be prosocial, unlike in previous studies, in which chimpanzees had to decide whether to reward a conspecific for no reason. Indeed, subjects' choices in the no-assistance condition reflected indifference, as in many other prosocial tasks, emphasizing that subjects' more prosocial behavior was not due to other differences in the procedure.

This raises a larger point. Often when animals fail to perform an expected behavior (or a human-typical behavior), we assume that it is because the behavior is not a common part of their repertoire or because they lack requisite cognitive abilities. Indeed, when experimenters try to find a context in which subjects do show a given behavior, they may be accused of digging for a result that they want, or a positive result that is eventually obtained may be considered overly context-dependent. Of course, these are real concerns; it is not good science to data mine or chase a result with no evidence for its existence. Nonetheless, when results conflict or experimental results do not match observations, we must remember that these results may be due to a failure by us to properly understand other animals' perspectives (de Waal, 2016). In their study, Schmelz et al. (2017) appear to have found the chimpanzees' perspective, and in so doing have provided both a deeper understanding of prosocial behavior in chimpanzees and a good reminder that our human perspective may bias our view of animal behavior and cognition.

\section{References}

Claidière, N., Whiten, A., Mareno, M. C., Messer, E. J. E., Brosnan, S. F., Hopper, L. M., . . . McGuigan, N. (2015). Selective and contagious prosocial resource donation in capuchin monkeys, chimpanzees and humans. Scientific Reports, 5, 7631. https://doi.org/10.1038/srep07631

de Waal, F. B. M. (2016). Are we smart enough to know how smart animals are? New York, NY: Norton.

Jensen, K., Hare, B., Call, J., \& Tomasello, M. (2006). What's in it for me? Self-regard precludes altruism and spite in chimpanzees. Proceedings of the Royal Society B, 273, 1013-1021. https://doi. org/10.1098/rspb.2005.3417

Schmelz, M., Grueneisen, S., Kabalak, A., Jost, J., \& Tomasello, M. (2017). Chimpanzees return favors at a personal cost. Proceedings of the National Academy of Sciences, 114, 7462-7467. https://doi. org/10.1073/pnas.1700351114

Silk, J. B., Brosnan, S. F., Vonk, J., Henrich, J., Povinelli, D. J., Richardson, A. S., . . . Schapiro, S. J. (2005). Chimpanzees are indifferent to the welfare of unrelated group members. Nature, 437, 1357-1359. https://doi.org/10.1038/nature04243 